\title{
INEQUALITIES FOR WEIGHTED SUMS OF POWERS AND THEIR APPLICATIONS
}

\author{
EDWARD NEUMAN
}

Abstract. Two inequalities for weighted sums of powers are established. Applications to Jacobian elliptic functions and Legendre's elliptic integrals of the first kind are presented. Some known and new inequalities for circular and hyperbolic functions are obtained. Applications to certain iterative means including Gauss' arithmetic-geometric mean and Schwab-Borchardt mean are included.

Mathematics subject classification (2010): Primary: 26D15, 33E05, 26D05, 26 D07.

Keywords and phrases: Inequalities, Jacobian elliptic functions, Legendre's elliptic integrals of the first kind, circular and hyperbolic functions, Gauss' arithmetic-geometric mean, Schwab-Borchardt mean.

\section{REFERENCES}

[1] J. M. Borwein, P. B. Borwein, Pi and AGM: A Study in Analytic Number Theory and Computational Complexity, John Wiley and Sons, New York, 1987.

[2] B. C. CARLson, Algorithms involving arithmetic and geometric means, Amer. Math. Monthly 78, (1971) 496-505.

[3] C. Huygens, Oeuvres Completes 1888-1840, Société Hollandaise des Science, Haga.

[4] D. F. Lawden, Elliptic Functions and Applications, Appl. Math. Sci., vol. 80, Springer-Verlag, New York, 1989.

[5] E. Neuman, One- and two-sided inequalities for Jacobian elliptic functions and related results, Integral Transforms Spec. Funct. 21 (2010), No. 6, 399-407.

[6] E. Neuman, On Wilker and Huygens type inequalities, Math. Inequal. Appl., 15, 2 (2012) 271-279.

[7] E. Neuman, Inequalities for the Schwab-Borchardt mean and their applications, J. Math. Inequal. 5 (2011), No. 4, 601-609.

[8] E. Neuman, J. SÁndor, On the Schwab-Borchardt mean, Math. Pannon. 14 (2003), No. 2, 253-266.

[9] E. Neuman, J. SÁndor, On the Schwab-Borchardt mean II, Math. Pannon. 17 (2006), No. 1, 49-59.

[10] E. NEUMAN, J. SÁNDOR, On some inequalities involving trigonometric and hyperbolic functions with emphasis on the Cusa-Huygens, Wilker and Huygens inequalities, Math. Inequal. Appl., 13 (2010), $715-723$

[11] F. W. J. Olver, D. W. Lozier, R. F. Boisvert, C. W. Clark, (EdS.), NiST Handbook of Mathematical Functions, Cambridge Univ. Press, New York, 2010.

[12] H.-J. SeIfFERT, Problem 887, Nieuw. Arch. Wisk. 11 (1993), 176.

[13] H.-J. SEIFFERT, Aufgabe 16, Wurzel 29 (1995), 87.

[14] E. T. Whittaker, G. N. Watson, A Course in Modern Analysis, 4th ed., Cambridge Univ. Press, New York, 1990.

[15] J. B. Wilker, Problem E 3306, Amer. Math. Monthly 96 (1989), 55.

[16] S.-H. WU AND H. M. SRIVASTAVA, A weighted and exponential generalization of Wilker's inequality and its applications, Integral Transforms and Spec. Funct. 18 (2007), No. 8, 529-535.

[17] S. WU AND A. BARICZ, Generalizations of Mitrinović, Adamović, and Lazarević's inequalities and their applications, Publ. Math. Debrecen 75/3-4 (2009), 447-458. 